\title{
Photoionization cross sections of O II, O III, O IV, and O V: Benchmarking $R$-matrix theory and experiments
}

\author{
Sultana N. Nahar \\ Department of Astronomy, The Ohio State University, Columbus, Ohio 43210, USA
}

(Received 16 August 2003; published 19 April 2004)

\begin{abstract}
For crucial tests between theory and experiment, $a b$ initio close-coupling calculations are carried out for photoionization of several oxygen ions: $\mathrm{O}$ II, O III, O IV, and O v. The relativistic fine-structure and resonance effects are studied using the $R$ matrix and its relativistic variant, the Breit-Pauli $R$-matrix (BPRM) approximations. Extremely detailed comparison is made with high-resolution experimental measurements carried out in three different setups: Advanced Light Source at Berkeley, synchrotron radiation experiment with a new modulator at University of Aarhüs, and synchrotron radiation experiment at University of Paris-Sud. The comparisons illustrate physical effects in photoionization such as (i) fine structure, (ii) resolution, and (iii) metastable components. Photoionization cross sections $\sigma_{P I}$ of the ground and a few low-lying excited states of these ions obtained in the experimental spectrum include combined features of these states. The general features of the measured $\sigma_{P I}$ were predicted in the earlier calculations in $L S$ coupling. However, due to higher-resolution achievement in the recent experiments, the theoretically calculated resonances need to be resolved with much finer energy mesh for precise comparison. In addition, prominent resonant features are observed in the measured spectra that can form from transitions allowed with relativistic fine structure, but not in $L S$ coupling. Present results include calculations from both $L S$ coupling and the relativistic BPRM method to decipher the features in photoionization spectra. $\sigma_{P I}$ are obtained for ground and metastable (i) $2 s^{2} 2 p^{3}\left({ }^{4} S^{o},{ }^{2} D^{o},{ }^{2} P^{o}\right)$ states of O II, (ii) $2 s^{2} 2 p^{2}\left({ }^{3} P,{ }^{1} D,{ }^{1} S\right)$ and $2 s 2 p^{3}\left({ }^{5} S^{o}\right)$ states of O III, (iii) $2 s^{2} 2 p\left({ }^{2} P_{J}^{o}\right)$ and $2 s 2 p^{2}\left({ }^{4} P_{J}\right)$ levels of $\mathrm{O}$ IV , and (iv) $2 s^{2}\left({ }^{1} S\right)$ and $2 s 2 p\left({ }^{3} P^{o},{ }^{1} P^{o}\right)$ states of $\mathrm{O} \mathrm{v}$. Most of the computed theoretical resonance structures are seen experimentally, although there are some discrepancies. One of the main conclusions of the present study is that resonances in photoionization cross sections of the ground and metastable states can be a diagnostic of experimental beam composition, with potential applications to astrophysical and laboratory plasma environments.
\end{abstract}

DOI: 10.1103/PhysRevA.69.042714

PACS number(s): $32.80 . \mathrm{Fb}$

\section{INTRODUCTION}

Photoionization cross sections of many atoms and ions were studied for the first time in detail with resonance structures for ground and many excited states under the Opacity Project (OP) [1]. The close-coupling (CC) $R$-matrix method, as employed under the OP, accurately considers the numerous autoionizing resonances in cross sections along interacting (overlapping) Rydberg series. The study under the OP resulted in new features in the cross sections, such as prominent photoexcitation of core resonances. The radiative work under the OP has been extended to collisional and radiative processes under the Iron Project (IP) [2] with inclusion of relativistic effects using the Briet-Pauli $R$-matrix (BPRM) method. However, while the methods used are state of the art, possible deficiencies are (i) the resonance structures in the OP/IP cross sections are not always fully resolved and (ii) the important near-threshold resonances may not be accurate owing to inadequate eigenfunction expansions. A prime need therefore is to benchmark the vast amount of theoretical OP/IP data that are now being utilized for global applications such as calculation of stellar opacities and photoionization rates in astrophysical models.

On the experimental side, accurate photoionization cross sections are being measured at several places, such as the merged ion-photon beam facility at the University of Aarhüs (e.g., for C II [3]), the Electron Cyclotron Resonant Ion
Source (ECR) at the University of Paris-Sud (e.g., for Xe V-Xe VIII [4]), and the Advanced Light Source (ALS) at Berkeley (e.g., for O II [5]). Each of these three sophisticated experimental setups has different advantages in measurements, and the results often complement each other. Cross sections for $\mathrm{O}$ II have been measured at ALS for nearthreshold features in $\sigma_{P I}$ of metastable states [5], and later extended to a wider photon energy range [6], and at the University of Aarhüs [7]. Detailed photoionization cross sections have recently been measured for the ground and low excited states of multiply charged oxygen ions for the first time for $\mathrm{O}$ III, O IV, and $\mathrm{OV}$, at the synchrotron radiation source at University of Paris-Sud [8]. Study of oxygen ions is of great importance because of their abundances in terrestrial and planetary atmospheres and in astrophysical objects.

Comparison of all these theoretical and experimental results is extremely crucial to evaluate the accuracy of both the theory and experiment, and to study the resonance phenomena. Theoretical work can also provide spectroscopic identification of resonances, and a quantitative measure of the mixture of states in the experimental beam. The resonant structures and the background shapes of the recently measured photoionization cross sections of oxygen ions are found to be in close agreement overall with some earlier theoretical cross sections [9]. However, certain features in the measured cross sections are not seen prominently or are missing in the calculated cross sections. 
One of the aims of this work is to study relativistic effects and fine-structure. We distinguish these in terms of relativistic mixing and fine-structure splitting with reference to pure $L S$ coupling results. While relativistic effects per se might not be of great importance for these light ions, fine-structure splitting manifests itself in many experimentally observed features. In addition, relativistic mixing via $E 1$ transitions (e.g., singlet-triplet and doublet-quartet) results in additional resonances not allowed in $L S$ coupling. The present study investigates the $L S$ coupling and fine-structure effects together with resonance phenomena in detail. Thus to begin with it is necessary to do $L S$ coupling calculations at high resolution. Comparison with experiment then reveals whether fine structure is also to be considered in order to analyze experimental data. In such cases the relativistic BPRM method is employed to compute cross sections.

Detailed comparison of experimental and theoretical resonance positions and shapes also highlights the importance of correlation effects in theoretical calculations. Resonances may be shifted with respect to their respective thresholds of convergence, indicating missing correlation configurations in the wave-function expansion. This again is crucial since the inherent accuracy of the close-coupling calculations depends on the completeness of the eigenfunction expansions, in particular, short-range correlation at low energies.

Another motivation for these studies is that the $R$-matrix method, as developed for the OP/IP work, has been extended to a theoretically self-consistent treatment of photoionization and unified electron-ion recombination including radiative and dielectronic recombination in an $a b$ initio manner [10-12]. The total recombination cross sections have been benchmarked for accuracy by comparing with several experimental cross sections $[11,12]$. However, while recombination cross sections require total contributions of photorecombination cross sections from all bound levels, obtained from the corresponding $\sigma_{P I}$ using detailed balance, comparison of photoionization cross sections with experiment involves individual features of $\sigma_{P I}$ of single states. For instance, photoionization cross sections including relativistic effects in the close-coupling approximation, as developed under the IP, was first benchmarked by comparing with the measured $\sigma_{P I}$ of C II [13].

The theory and calculations are outlined in Secs. II and III, respectively, followed by a discussion of results for the four ions $\mathrm{O}$ II-O V in Sec. IV, and the conclusions in Sec. V.

\section{THEORY}

Present calculations are carried out in an ab initio manner in the close-coupling $R$-matrix method (e.g., Refs. [14,15]) as employed in the Opacity Project [1] and the Iron Project [2]. The package of codes from the OP [15] have been extended to include relativistic effects in the Breit-Pauli approximation in the IP work $[2,16,17]$ under the name BreitPauli $R$-matrix method. A brief discussion of the method is given below.

In the close-coupling approximation the total wave function of the $(N+1)$-electron ion is represented by the wave functions of the $N$-electron core, multiplied by the wave function of the outer electron:

$$
\Psi_{E}(e+\text { ion })=A \sum_{i} \chi_{i}(\text { ion }) \theta_{i}+\sum_{j} c_{j} \Phi_{j}(e+\text { ion })
$$

where $\chi_{i}$ is the target (or the core) wave function in a specific state $S_{i} L_{i} \pi_{i}$ or level $J_{i} \pi_{i}$ and $\theta_{i}$ is the wave function for the $(N+1)$ th electron in a channel labeled $S_{i} L_{i}\left(J_{i}\right) \pi_{i} k_{i}^{2} l_{i} S L(J \pi) ; k_{i}^{2}$ is its incident kinetic electron energy. $\Phi_{j}$ 's are the correlation functions of the $(N+1)$-electron system that account for short-range correlation and the orthogonality between the continuum and the bound orbitals. The complex resonant structures in photoionization are included through channel couplings.

Relativistic effects are included through Breit-Pauli approximation in intermediate coupling. The Breit-Pauli Hamiltonian, as adopted in the IP work [2], has the following terms:

$$
H_{N+1}^{\mathrm{BP}}=H_{N+1}+H_{N+1}^{\mathrm{mass}}+H_{N+1}^{\mathrm{Dar}}+H_{N+1}^{\mathrm{so}},
$$

where $H_{N+1}$ is the nonrelativistic Hamiltonian,

$$
H_{N+1}=\sum_{i=1}^{N+1}\left\{-\nabla_{i}^{2}-\frac{2 Z}{r_{i}}+\sum_{j>i}^{N+1} \frac{2}{r_{i j}}\right\},
$$

and the additional terms represent the one-body mass correction, the Darwin, and the spin-orbit interaction terms, respectively,

$$
\begin{gathered}
H_{N+1}^{\mathrm{mass}}=-\frac{\alpha^{2}}{4} \sum_{i} p_{i}^{4}, \\
H_{N+1}^{\mathrm{Dar}}=\frac{Z \alpha^{2}}{4} \sum_{i} \nabla^{2}\left(\frac{1}{r_{i}}\right), \quad H_{N+1}^{\mathrm{so}}=Z \alpha^{2} \sum_{i} \frac{1}{r_{i}^{3}} \mathbf{l}_{\mathrm{i}} \cdot \mathbf{s}_{\mathrm{i}} .
\end{gathered}
$$

The set of $S L \pi$ are recoupled to obtain $(e+$ ion $)$ states with total $J \pi$, following the diagonalization of the $(N+1)$-electron Hamiltonian to solve

$$
H_{N+1}^{B P} \Psi_{E}=E \Psi_{E} .
$$

Substitution of the wave-function expansion introduces a set of coupled equations that are solved using the $R$-matrix approach. The continuum wave function $\Psi_{F}$ describe the scattering process with the free electron interacting with the target at positive energies $(E>0)$, while the solutions correspond to pure bound states $\Psi_{B}$ at negative total energies $(E<0)$.

The photoionization cross section $\sigma_{P I}$ is proportional to the generalized line strength $S$,

$$
\sigma_{P I}=\frac{4 \pi}{3 c} \frac{1}{g_{i}} \omega S
$$

where $S$ is the generalized line strength,

$$
S_{\mathrm{L}}=\left|\left\langle\Psi_{j}\left\|\mathbf{D}_{L}\right\| \Psi_{i}\right\rangle\right|^{2},
$$

$\mathbf{D}$ is the dipole operator, $\mathbf{D}_{L}=\Sigma_{i} r_{i}$ in "length" form, and $\mathbf{D}_{V}$ $=-2 \sum_{i} \Delta_{i}$ in "velocity" form, where the sum corresponds to number of electrons. 


\section{CAlCUlations}

Photoionization cross sections of O II, O III, O IV, and O V are obtained using the same wave-function expansions for residual core ions as developed in earlier work (Nahar [9]). Calculations are carried out both in nonrelativistic $L S$ coupling and in the BPRM approximation.

The wave functions of the target or core ion were obtained from atomic structure calculations using a scaled Thomas-Fermi model in the code SUPERSTRUCTURE [18]. The computation of $\mathrm{O}$ II photoionization cross sections is carried out with the wave-function expansion of 12 terms of O III of configurations $2 s^{2} 2 p^{2}, 2 s 2 p^{3}, 2 s^{2} 2 p 3 s$, and $2 p^{4}$ as in Ref. [9]. The wave-function expansion for O III consists of configurations $2 s^{2} 2 p, 2 s 2 p^{2}, 2 p^{3}, 2 s^{2} 3 s, 2 s^{2} 3 p, 2 s^{2} 3 d$, $2 s 2 p 3 s$, and $2 s 2 p 3 p$ of O IV. However, to include the fine structure in calculating $\sigma_{P I}$ for $2 s 2 p^{3}\left({ }^{5} S^{o}\right)$ state of $\mathrm{O}$ III, an expansion of 15 levels of O IV with configurations $2 s^{2} 2 p$, $2 s 2 p^{2}$, and $2 p^{3}$ was used. The O IV eigenfunctions consist of a 20-level expansion of the core ion $\mathrm{O} \mathrm{V}$ with configurations $2 s^{2}, 2 s 2 p, 2 p^{2}, 2 s 3 s, 2 s 3 p$, and $2 s 3 d$. The wave-function expansion for $\mathrm{O} v$ consists of nine states of the core ion $\mathrm{O}$ VI with $1 s^{2} 2 s, 1 s^{2} 2 p, 1 s^{2} 3 s, 1 s^{2} 3 p, 1 s^{2} 3 d, 1 s^{2} 4 s, 1 s^{2} 4 p, 1 s^{2} 4 d$, and $1 s^{2} 4 f$. The calculated target level energies were replaced by the slightly different observed energies to obtain somewhat more accurate positions for the resonances that belong to Rydberg series converging onto the target thresholds.

The short-range bound channel correlation term in the $\mathrm{CC}$ expansion, the second term in Eq. (2.1), includes all possible $(N+1)$-electron configurations as in Ref. [9] for $L S$ coupling calculations. BPRM calculations are carried out for $\mathrm{O}$ III and $\mathrm{O}$ IV to elicit fine-structure details apparent in experimental data. For the BPRM calculations of $\mathrm{O}$ III, all configurations are considered with maximum orbital occupancies $2 s^{2}, 2 p^{4}$, $3 s^{2}, 3 p^{2}$, and $3 d^{2}$, while the $K$-shell remains filled. For O IV, the $(N+1)$-electron configurations are up to $1 s^{2}, 2 p^{3}, 3 s^{2}$, $3 p^{2}, 3 d^{2}, 4 s^{2}$, and $4 p^{2}$, while $K$-shell may be open with a single occupancy.

Computations of $\sigma_{P I}$ were carried out using the package of BPRM codes from the Iron Project [17]. Present calculations, aiming to compare with the experiments, are considerably larger because of inclusion of fine structure and repetition of computation many times, especially for search of narrow resonant structures. Cross sections in small energy bins were scanned with variable energy meshes and delineated with finer energy meshes, ranging from $10^{-5}$ to $10^{-7}$ Ry. While measurement includes detection of features within the experimental monochromatic bandwidth, calculations show features only at calculated points. Hence, a narrow resonance may show up in the measurement in an averaged convolved form, but missed in the calculations if the energy mesh is not fine for the width of the resonance. For complex ions, resonances are extensive and overlapping, and are easy to miss when energy mesh is not fine enough. A direct comparison of theoretical and experimental resonant structures may not be possible if the resonances are narrower than the monochromatic bandwidth of the experimental beam.

For comparison with experiment, the resonances are convolved with a Gaussian distribution function with full width half maximum (FWHM) equal to the energy bandwidth of the monochrometer in the experiment $(35 \mathrm{meV}$ at Aahus University set up, $17 \mathrm{meV}$ at ALS, and 20-30 meV at University of Paris setup). The average cross section $\sigma_{P I}^{a v}\left(E_{i}\right)$ at energy $E_{i}$ convoluted over the FWHM, $E_{o}$, or the monochromatic bandwidth, is obtained as

$$
\sigma_{P I}^{a v}\left(E_{i}\right)=\int_{E_{i}-E_{o} / 2}^{E_{i}+E_{o} / 2} \sigma_{P I}(E) \frac{\exp \left(-x^{2}\right)}{\sqrt{\pi} E_{o}} d E,
$$

where $x=\left(E-E_{i}\right) / E_{o}$. Present cross sections have been convolved with typical energy bandwidths used in various experiments for comparison. The photon energies are shifted slightly to match the measured ionization threshold of each state exactly.

\section{RESULTS AND DISCUSSION}

Photoionization cross sections of the ground and several lowest excited states of O II, O III, O IV, and O V are obtained and compared with the recent precise and high-resolution measurements at the ALS at Berkeley, the University of Aarhüs, and the University of Paris-Sud. The resonances of detailed photoionization cross sections have been convolved with experimental bandwidth, as mentioned above, using a Gaussian distribution function to compare with the measured cross sections. Both the detailed and convolved $\sigma_{P I}$ are presented for each ion.

Photoionization cross sections in $L S$ coupling in, general, show very good agreement with experiment, indicating that relativistic effects are not significant for most states considered, especially for $\mathrm{O}$ II and $\mathrm{O}$ v. However, inclusion of relativistic effects is crucial for some metastable states for O III and $\mathrm{O}$ IV.

The main objective of this paper is to compare the theory and experiment for the existence and the positions of resonant structures and their identification in the cross sections. However, the exact shape and peak of the resonances, as well as the background cross sections, depend on (i) resolution, (ii) distribution function for the beam shape, and (iii) fractions or mixture of states in the beam. The third point can be difficult to determine exactly as the beam composition may vary with energy during measurements. With some numerical variations of abundances and beamwidth, the theoretical and experimental shapes can be brought in closer agreement, which however is not the main emphasis of the present work. The Gaussian distribution function, which peaks around the center, is used for convolution of theoretical cross sections. This may not describe exactly the distribution function of the experimental beam. Experimental calibration of the residual ion threshold energies is also an important factor for locating resonance positions.

Observed resonant features in photoionization of oxygen ions have been identified in Refs. [5-8]. Resonances can be identified using quantum defect analysis $E_{r}=E_{t}-z^{2} / \nu^{2}$, where $E_{r}$ is the resonance energy, $E_{t}$ is the next core threshold energy in rydbergs, $z=Z-n_{c}$ is the ion charge of the target where $n_{c}$ is the number of core electrons, and $\nu=n$ $-\mu$ is the effective quantum number with $\mu$ being the quantum defect. $\mu$ is large for low angular momenta, such as for 


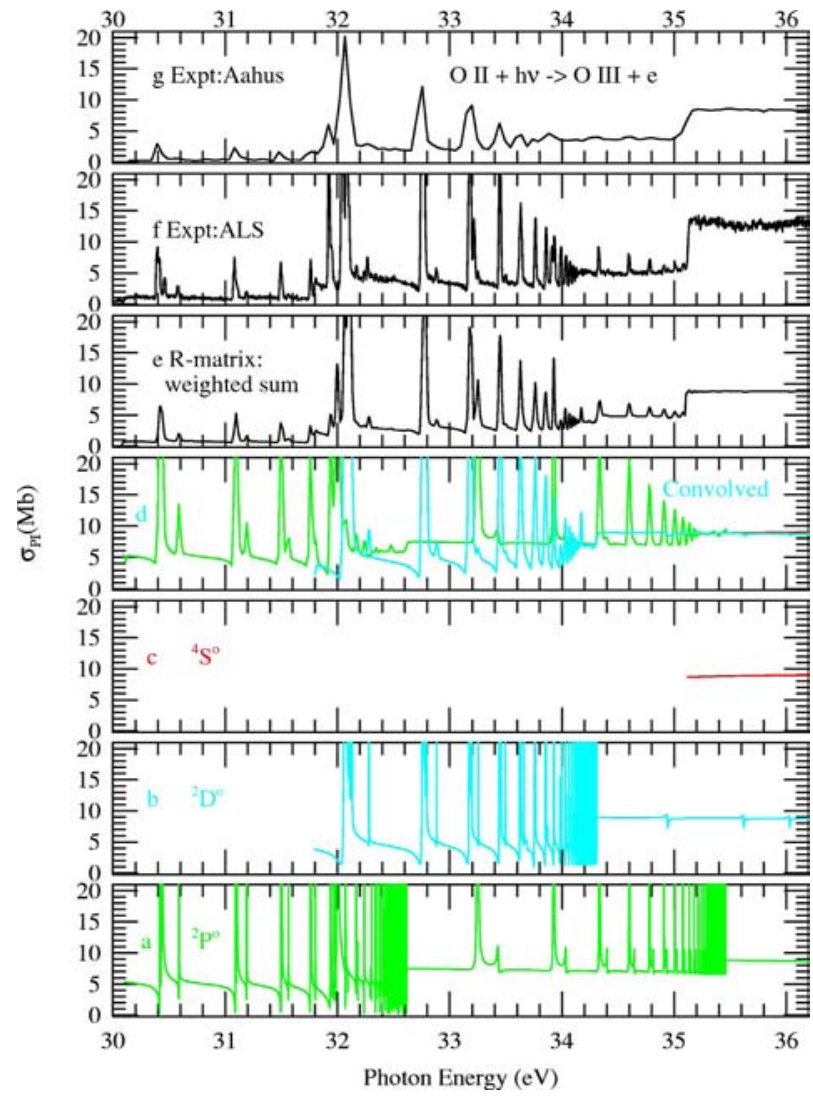

FIG. 1. (Color online) Photoionization cross sections $\sigma_{P I}$ of ground ${ }^{4} S^{o}$ and metastable ${ }^{2} P^{o},{ }^{2} D^{o}$ states of $\mathrm{O}$ II in the low-energy region, 30-36 eV: panels (a), (b), and (c), detailed $\sigma_{P I}$ of ${ }^{2} P^{o}$ (green), ${ }^{2} D^{o}$ (cyan), and ${ }^{4} S^{o}$ (red) states, respectively; (d) the same cross sections, but convolved with experimental monochrometer bandwidth; (e) sum of the convolved cross sections weighted by experimental beam mixtures to compare with measured $\sigma_{P I}$; (f), (g), measured $\sigma_{P I}$ by Covington et al. [5] and by Kjeldsen et al. [7], respectively.

an $s$ electron, and decreases with higher angular momenta approaching near zero for $d$ orbitals in oxygen ions.

The BPRM results have many more resonances than in $L S$ coupling due to (i) an increment in number of Rydberg series of resonances with increased number of core thresholds with fine structure and (ii) relativistic mixing of $E 1$ allowed and intercombination transitions. $L S$ coupling results are approximately the statistical average over the fine structure and does not consider intercombination transitions.

Results for each ion are presented and discussed in separate sections below.

\section{A. Photoionization of $\mathrm{O}$ II}

Photoionization cross sections of the ground ${ }^{4} S^{o}$ state and the metastable ${ }^{2} \mathrm{P}^{o},{ }^{2} \mathrm{D}^{o}$ states of $\mathrm{O}$ II in the low-energy region, 30-36 eV, are shown in Fig. 1. The figure has seven panels: the lowest three panels, Figs. 1(a)-1(c), present the detailed $\sigma_{P I}$ of ${ }^{4} S^{o}$ (red curve), ${ }^{2} D^{o}$ (cyan), ${ }^{2} P^{o}$ (green) states, respectively. These cross sections include extensive resonances due to Rydberg series of autoionizing states. They become extremely narrow as the autoionizing states converge onto the core thresholds, ${ }^{1} D,{ }^{1} P,{ }^{5} S^{o}$, etc., and their integrated strength is usually small. They are wiped out during convolution, and are not observed, as can be seen in the convolved cross sections of ${ }^{4} S^{o},{ }^{2} D^{o}$, and ${ }^{2} P^{o}$ states in panel (d). The colors of curves identify the resonances of the states they belong to. Panel (e) presents sums of the convolved cross sections with mixing fractions $15 \%$ for ${ }^{2} P^{o}, 42 \%$ for ${ }^{2} D^{o}$, and $43 \%$ for ${ }^{4} S^{o}$, as deduced from experimental beam mixture in the ALS experiment. The summed cross sections can be compared directly with the $\sigma_{P I}$ measured at ALS [panel (f)] and at Aarhüs [panel (g)]. Although the mixing proportions are somewhat different in the two experiments the prominent features appear in both. The ALS cross sections have much higher resolution $(17 \mathrm{meV})$ than those from Aarhüs (35 meV) because of the much narrower bandwidth. However, the ALS values are relative while the Aarhüs cross sections are absolute. Very good agreement can be seen between the theoretical and experimental features. The most significant point is that all the resonant features in the observed photoionization spectra have now been found in the calculated cross sections. Previous calculations [9] used much coarser energy mesh in calculating $\sigma_{P I}$ and missed some resonances while others were not adequately resolved.

Figure 2 presents $\sigma_{P I}$ of the same states, the ground ${ }^{4} S^{o}$ and the metastable ${ }^{2} P^{o},{ }^{2} D^{o}$, but in the high-energy region, 36-46 eV. It also has seven panels: the lowest three panels (a,b,c) present the detailed $\sigma_{P I}$ of ${ }^{4} S^{o}$ (red curve), ${ }^{2} D^{o}$ (cyan), ${ }^{2} P^{o}$ (green) states, respectively. The extensive narrow resonant structures of highly excited Rydberg states converging onto the various core thresholds are nearly wiped out during convolution, as seen in the convolved cross sections for ${ }^{4} S^{o}$, ${ }^{2} D^{o}$, and ${ }^{2} P^{o}$ states together in panel (d). Identical colors are used for both the detailed and the convolved cross sections of each state. Panel (e) presents sum of the convolved $\sigma_{P I}$ with mixtures of $15 \%$ for ${ }^{2} P^{o}, 42 \%$ for ${ }^{2} D^{o}$, and $43 \%$ for ${ }^{4} S^{o}$ as in the ALS experimental beam. The summed cross sections are compared with the measured $\sigma_{P I}$ at ALS [panel (f)] and Aarhüs [panel $(\mathrm{g})$ ]. Very good agreement can be seen for most the of features theoretically and experimentally. However, some discrepancies are noted. The observed resonance at $37.5 \mathrm{eV}$, belonging to ${ }^{2} D^{o}$ state, is shifted to the right (higher energy) by about $0.5 \mathrm{eV}$ in the calculated spectrum. This may have resulted from some missing correlation configurations. Both the experiments and the theory show the ${ }^{2} D^{o}$ resonance at $38.9 \mathrm{eV}$. But the two resonances below this, at about 38.4 and $38.7 \mathrm{eV}$ found in ALS spectrum, are not seen in the calculated cross sections. It is possible that they belong to some other states in the mixture.

The resonance series have been identified in Refs. [5-7]. For example, the resonances below $31.8 \mathrm{eV}$ (threshold energy for $\left.{ }^{2} D^{o}\right)$ in Fig. 1 are from $2 p^{3}\left({ }^{2} P^{o}\right)$ state which can photoionize to ${ }^{2} S,{ }^{2} P$, and ${ }^{2} D$ states with $2 p$ electron going to $\epsilon d$ or $\epsilon s$ continua. Hence, the resonances belong to $2 p^{2}\left({ }^{1} D\right) n d\left({ }^{2} S,{ }^{2} P,{ }^{2} D\right)$ and $2 p^{2}\left({ }^{1} D\right) n s\left({ }^{2} D\right)$ Rydberg series of threshold $2 p^{2}\left({ }^{1} D\right)$. Resonances with higher angularmomentum transition, $p \rightarrow d$, in general are more prominent than those with the lower one, $p \rightarrow s$. Hence, the $n d$ series can be easily differentiated from $n s$ series via quantum defects and similarity in features. For example, of the two dis- 


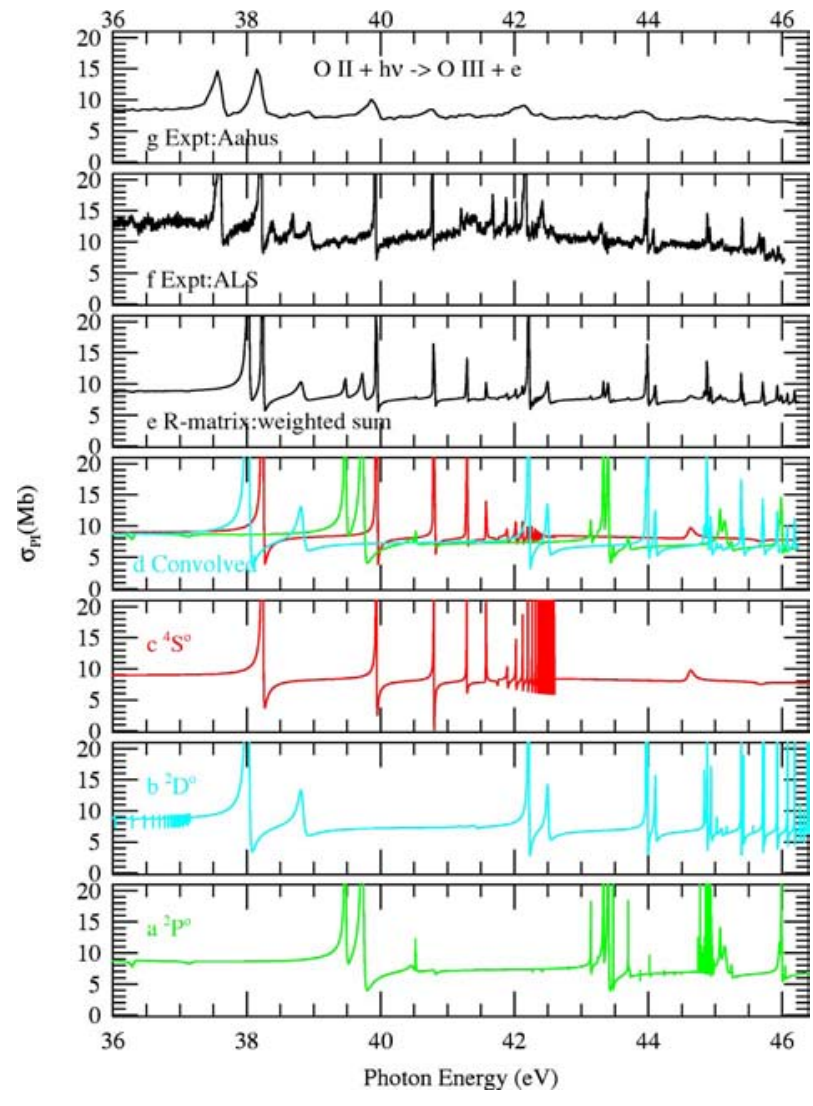

FIG. 2. (Color online) Photoionization cross sections $\sigma_{P I}$ of ground ${ }^{4} S^{o}$ and metastable ${ }^{2} P^{o},{ }^{2} D^{o}$ states of $\mathrm{O}$ II in the higherenergy region. 36-46 eV: panels (a), (b), and (c), detailed $\sigma_{P I}$ of ${ }^{2} P^{o}$ (green), ${ }^{2} D^{o}$ (cyan), and ${ }^{4} S^{o}$ (red) states, respectively; (d) the same cross sections, but convolved with experimental monochrometer bandwidth; (e) sum of the convolved cross sections weighted by experimental beam mixtures to compare with measured $\sigma_{P I}$; (f),(g), measured $\sigma_{P I}$ by Aguilar et al. [6] and by Kjeldsen et al. [7], respectively.

tinct resonance series seen in the low-energy region of Fig. 1(d), the first series (with higher peaks) actually represents three overlapping nd series of resonances, $2 p^{2}\left({ }^{1} D\right) n d\left({ }^{2} S,{ }^{2} P,{ }^{2} D\right)$, and the second one (lower peaks) is the $n s$ series, $2 p^{2}\left({ }^{1} D\right) n s\left({ }^{2} D\right)$. The table of identified resonances is given in Refs. [5,7].

\section{B. Photoionization of $\mathrm{O}$ III}

The first measurement of photoionization cross sections for O III is reported by the University of Paris-Sud [8]. To compare with the experiment, $\sigma_{P I}$ are obtained with high resolution of resonances for the three lowest states, $2 s^{2} 2 p^{2}\left({ }^{3} P,{ }^{1} D,{ }^{1} S\right)$ of the ground configuration, and the metastable $2 s 2 p^{3}\left({ }^{5} S^{o}\right)$ state of $\mathrm{O}$ III using the $23 \mathrm{CC}$ wavefunction expansion of Nahar [9]. The detailed cross sections of the three states, $2 s^{2} 2 p^{2}\left({ }^{3} P,{ }^{1} D,{ }^{1} S\right)$, are presented in panels [Figs. 3(a)-3(c)], $\left({ }^{3} P\right.$-red, ${ }^{1} D$-blue, and ${ }^{1} S$-green) and the metastable ${ }^{5} S^{o}$ state in Fig. 3(d) (pink). The convolved cross sections are presented in panel (e) and the weighted sum in panel (f). Present detailed cross sections with higher

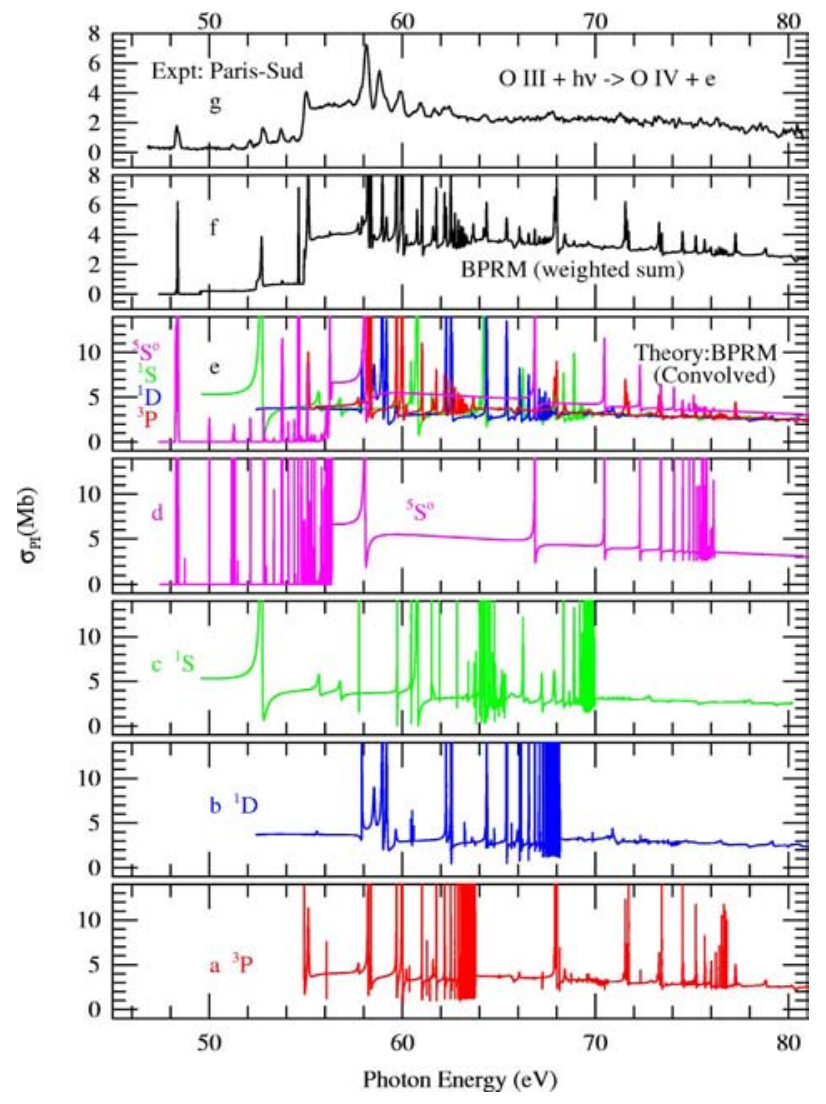

FIG. 3. (Color online) Photoionization cross sections $\sigma_{P I}$ of O III: panels (a), (b), (c), $2 s^{2} 2 p^{2}\left({ }^{3} P,{ }^{1} D,{ }^{1} S\right)$ states; (d) $2 s 2 p^{3}\left({ }^{5} S^{o}\right)$; (e) convolved cross sections of ${ }^{3} P,{ }^{1} D,{ }^{1} S,{ }^{5} S^{o}$; (f) weighted sum of convolved cross sections; ( $g$ ) experimental cross sections of the combined states measured at University of Paris-Sud by Champeaux et al. [8].

resolution display much more narrow resonances compared to the earlier calculations.

Comparison between theory [panel (f)] and experiment [panel (g)] shows that all the resonances observed for the first three states are accounted for in the calculated values. However, while relativistic effects are not important to bring out the main features of the three $2 s^{2} 2 p^{2}\left({ }^{3} P,{ }^{1} D,{ }^{1} S\right)$ states, a closer examination of the experimental results shows that there are additional features, especially in the low-energy region [see panel $(\mathrm{g})$ ]. We therefore consider the first odd parity metastable state $2 s 2 p^{3}\left({ }^{5} S^{o}\right)$ of $\mathrm{O}$ III and include fine structure through BPRM calculations. Prominent resonant features are found in the low-energy region of $\sigma_{P I}$ of this state that are not allowed in $L S$ coupling, but are formed due to relativistic channel mixing. In particular, the very first resonance at $\sim 48 \mathrm{eV}$ in the experimentally observed cross sections is due to photoionization of the high-lying metastable $2 s 2 p^{3}\left({ }^{5} S^{o}\right)$.

The differences in $\sigma_{P I}$ between the $L S$ coupling and the BPRM fine-structure cross sections are outlined in Fig. 4. Figures 4(a) and 4(b) present $\sigma_{P I}\left({ }^{5} S^{o}\right)$ in $L S$ coupling and in the BPRM approximation, respectively. The cross sections in the two panels are very similar from a photon energy of $56.4 \mathrm{eV}$ onwards. Whereas $\sigma_{P I}$ is zero below this threshold 


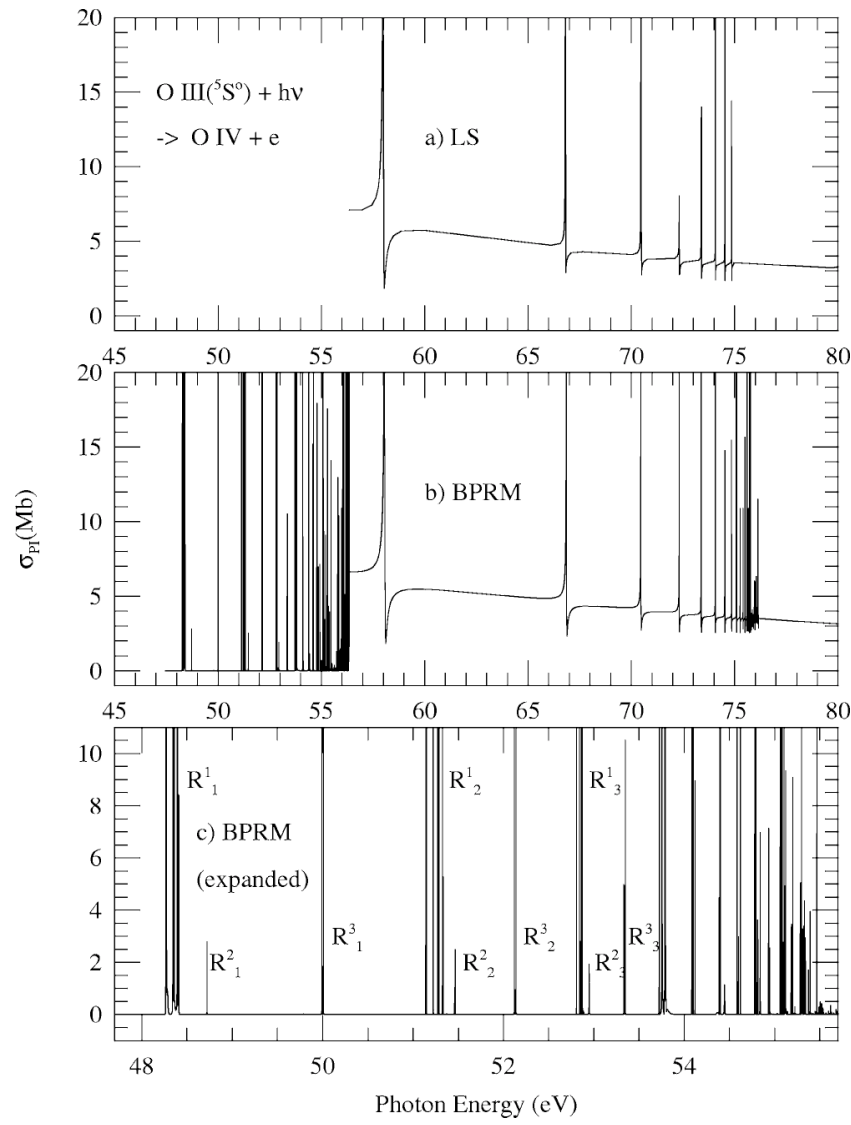

FIG. 4. Photoionization cross sections $\sigma_{P I}$ of the excited metastable $2 s 2 p^{3}\left({ }^{5} S_{2}^{o}\right)$ state of O III: panel (a), in $L S$ coupling; (b) in BPRM approximation; (c) $2 s 2 p^{2}{ }^{4} P n l(R)$ series identification in the low-energy region.

in $L S$ coupling, inclusion of relativistic effects in the BPRM calculations shows the region filled with extensive resonances with almost zero background cross section. At $56.4 \mathrm{eV}, 2 s 2 p^{3}\left({ }^{5} S^{o}\right)$ of $\mathrm{O}$ III ionizes leaving the core ion in the excited ${ }^{4} P$ state of $\mathrm{O}$ IV, which is the first threshold to the electron continuum. In $L S$ coupling, $2 s 2 p^{3}\left({ }^{5} S^{o}\right)$ state photoionizes only into a ${ }^{5} P(e+$ ion $)$ angular and spin symmetry. The ${ }^{5} P$ symmetry cannot be formed from the ground-state continuum of $\mathrm{O}$ IV, i.e., ${ }^{2} P^{o} n l$. In other words, $56.4 \mathrm{eV}$ is the ionization threshold for the ${ }^{5} S^{o}$ metastable state of O III in $L S$ coupling, and equals the sum of $47.4 \mathrm{eV}$, for ionization into the ground ${ }^{2} P^{o}$ state, and $8.85 \mathrm{eV}$, for excitation of the core to the $2 s 2 p^{2}\left({ }^{4} P\right)$ threshold. No features can be formed below this threshold in $L S$ coupling. However, fine structure allows ionization of the $2 s 2 p^{3}\left({ }^{5} S_{2}^{o}\right)$ level (with $J=2$ ) of O III into the core ground state of $\mathrm{O}$ IV through relativistic mixing, e.g., with ${ }^{2} P^{o} n p\left({ }^{3} P_{2}\right)$ level.

Expanded and detailed $\sigma_{P I}$ of $\mathrm{O}$ III below the ${ }^{4} P$ threshold of $\mathrm{O}$ IV are presented in Fig. 4(c) to enable comparison with experiment. Patterns of various resonance complexes, as denoted by $R^{n}$ series in the figure, can be discerned. These resonances belong to several Rydberg series of autoionizing levels, such as ${ }^{4} P_{1 / 2,3 / 2,5 / 2} n d\left({ }^{5} P_{J}\right)$ and ${ }^{4} P_{1 / 2,3 / 2,5 / 2} n s\left({ }^{5} P_{J}\right)$. The ${ }^{5} P_{J}$ levels are allowed to decay to ${ }^{2} P_{1 / 2,3 / 2}^{o} n p\left({ }^{1,3} P_{1,2}\right)$ levels via radiationless transitions. Decay to other levels, such as ${ }^{1,3} D_{1,2,3},{ }^{2,3} F_{1,3}$, are also possible through some relativistic mixing. However, these transitions are very weak.

The convolved cross sections with experimental monochromatic bandwidth for ${ }^{5} S^{o}$ state are shown in Fig. 3(e). Most of the computed resonances are difficult to observe in the experiment (top panel of Fig. 3) since their width is less than the monochromatic bandwidth of the detector in the experiment. A weighted sum of the ground and all metastable states included is given in Fig. 3(e). The excellent agreement between theory and experiment shows that the theoretical O III cross sections accurately reproduce the corresponding features in the experimental data.

\section{Photoionization cross sections of $\mathrm{O}$ IV}

Photoionization cross sections of O IV are presented for fine-structure levels of the ground state, $2 s^{2} 2 p\left({ }^{2} P_{1 / 2,3 / 2}^{o}\right)$, and of the first metastable state, $2 s 2 p^{2}\left({ }^{4} P_{1 / 2,3 / 2,5 / 2}\right)$. With increasing ion charge, and depending on the ionic species, fine structure can manifest itself more prominently which is the case for photoionization of boronlike O IV. Earlier works on $\mathrm{C}$ II, an isoelectronic partner of O IV, showed that some resonances observed in the experiment were missing from the $L S$ coupling results [3]. Both relativistic mixing and finestructure splitting were found to play a role in photoionization of B-like ions and explained the missing resonances [13].

Relativistic $\sigma_{P I}$ are presented for the levels ${ }^{2} P_{1 / 2,3 / 2}^{o}$ in Figs. 5(a) and 5(b) and ${ }^{4} P_{1 / 2,3 / 2,5 / 2}$ in Figs. 5(c)-5(e). Cross sections for the fine-structure levels of each term show very similar features. The number of resonances in the BPRM results is much larger in comparison to those in $L S$ coupling, as expected owing to fine-structure splitting of target thresholds resulting in a larger number of Rydberg series of resonances. For example, the $12 L S$ terms of the core ion $\mathrm{O} \mathrm{V}$ correspond to 20 fine-structure levels, which provide eight more series of resonances. Some additional resonances are also produced due to relativistic fine-structure couplings $(\Delta J=0, \pm 1)$ not allowed via $L S$ coupling.

Figures 5(c)-5(e) show prominent resonant features, although with almost zero background, in the low-energy BPRM cross sections of ${ }^{4} P$ levels. Extensive narrow resonances are seen to converge at about $78.7 \mathrm{eV}$, confirming enhancement in the observed photoionization cross section [Fig. 6(a) around this energy. Similar to the ${ }^{5} S^{o}$ state of O III, channel couplings of fine-structure levels are important in photoionization of the metastable ${ }^{4} \mathrm{P}$ term of $\mathrm{O}$ IV in forming these resonances. Without fine-structure mixing there will be no cross section below $78.7 \mathrm{eV}$ which is the ionization threshold for ${ }^{4} P$ state in $L S$ coupling and photoionization will occur by leaving the core $\mathrm{OV}$ in ${ }^{3} P^{o}$ state as it is coupled to ${ }^{4} P$ state of O IV. At this energy, both the BPRM and $L S$ coupling calculations give the same photoionization cross sections (analogous to the ${ }^{5} S^{o}$ state of O III). However, couplings of fine-structure levels lowers the ionization threshold from $78.7 \mathrm{eV}$ by about $10.2 \mathrm{eV}$ to $68.5 \mathrm{eV}$, where $10.2 \mathrm{eV}$ is the energy difference between the target thresholds $2 s^{2}\left({ }^{1} S\right)$ and $2 s 2 p\left({ }^{3} P^{o}\right)$ of O v. These low-energy resonances can be explained as follows. The $2 s 2 p^{2}{ }^{4} P$ state can photoionize to ${ }^{4} D^{o},{ }^{4} P^{o},{ }^{4} S^{o} S L \pi$ symmetries of the (e 


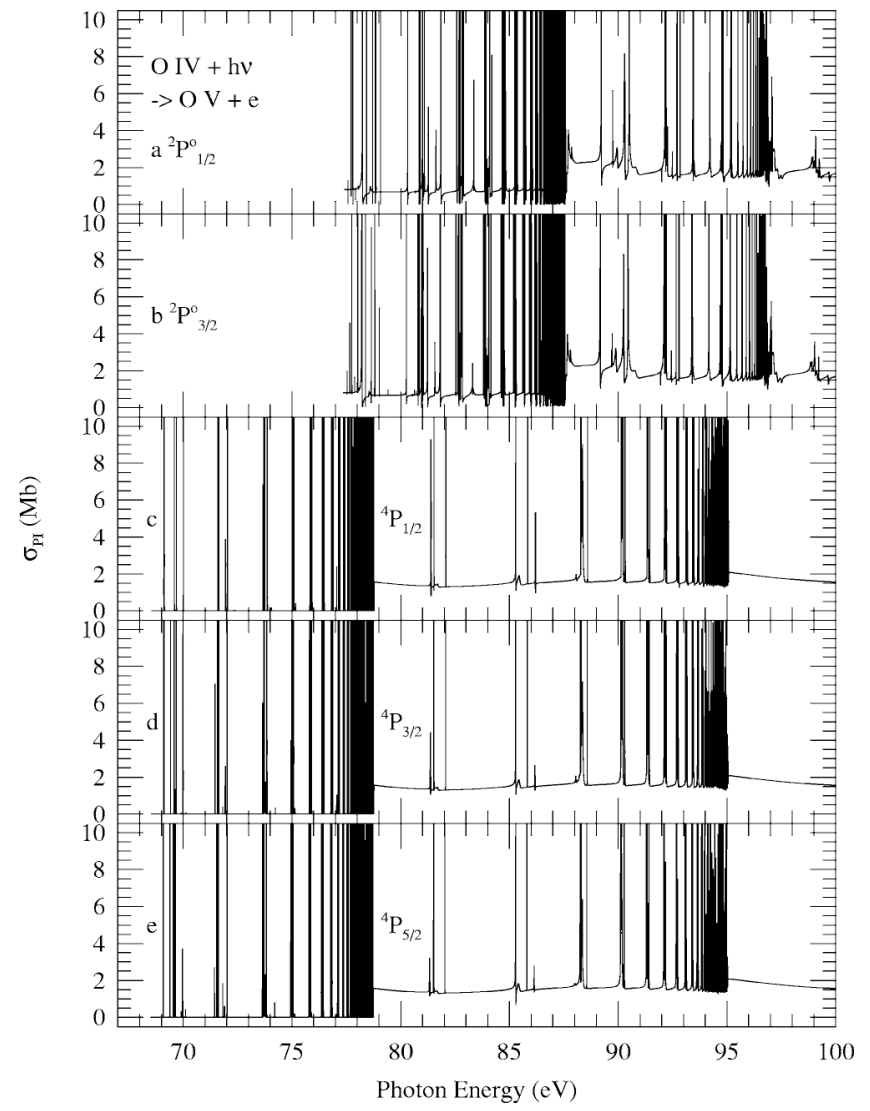

FIG. 5. Photoionization cross sections $\sigma_{P I}$ of O IV in the BPRM approximation: (a),(b) ${ }^{2} P_{1 / 2,3 / 2}^{o}$ levels of ground configuration, $2 s^{2} 2 p$; (c),(d),(f) excited $2 s 2 p^{2}\left({ }^{4} P_{1 / 2,3 / 2,5 / 2}\right)$ levels.

+ion) continua. Interaction of channels with fine structure allows couplings of these states with total angular momenta (SL) $J \pi \quad J \pi=1 / 2^{\circ}$ and $3 / 2^{\circ}$. The autoionizing levels $2 s 2 p\left({ }^{3} P^{o}\right) n d\left({ }^{4} D^{o}\right)$ thereby decay to $J \pi=1 / 2^{o}$ and $3 / 2^{o}$ continua of $2 s^{2}\left({ }^{1} S\right) \epsilon p\left({ }^{2} P^{o}\right)$ via radiationless transitions.

To compare these photoionization cross sections directly with the experimentally measured ones, the cross sections are convolved using a Gaussian distribution function with FWHM equal to the experimental monochromatic beamwidth. $\sigma_{P I}$ of each level are then statistically averaged as $\left[\Sigma_{i}\left(2 S_{i}+1\right)\left(2 L_{i}+1\right) \sigma_{P I} / \Sigma_{i}\left(2 S_{i}+1\right)\left(2 L_{i}+1\right)\right]$ to obtain the convolved cross sections of ${ }^{2} P^{o}$ and ${ }^{4} P$ states of O IV. These convolved and averaged $\sigma_{P I}$ are presented in Fig. 6(c) (red${ }^{2} P^{o}$ and green $\left.-{ }^{4} P\right) . \sigma_{P I}\left({ }^{4} P\right)$ are multiplied by $9 \%$ and $\sigma_{P I}^{2} P^{o}$ by $91 \%$ before addition to obtain the weighted sum $\sigma_{P I}$. These weighted cross sections are given in Fig. 6(b). The top panel presents the experimentally measured photoionization cross sections at the University of Paris-Sud [8].

Resonances in the energy region below the ground ${ }^{2} \mathrm{P}^{o}$ ionization threshold, $77.4 \mathrm{eV}$ (Fig. 6), are due to the metastable ${ }^{4} P$ state [green curve in Fig. 6(c)]. The metastable state is relatively easy to excite as it corresponds to a $\Delta n$ $=0$ transition $\left(2 s^{2} 2 p-2 s 2 p^{2}\right)$ and its features are observed. The first resonance in Fig. 6 at about $69.7 \mathrm{eV}$ is confirmed by both theory and experiment. However, some shift in theoretical positions (by $\sim 0.5 \mathrm{eV}$ ) can be seen for the next three resonances. The exact reason for this shift is not clear, but a

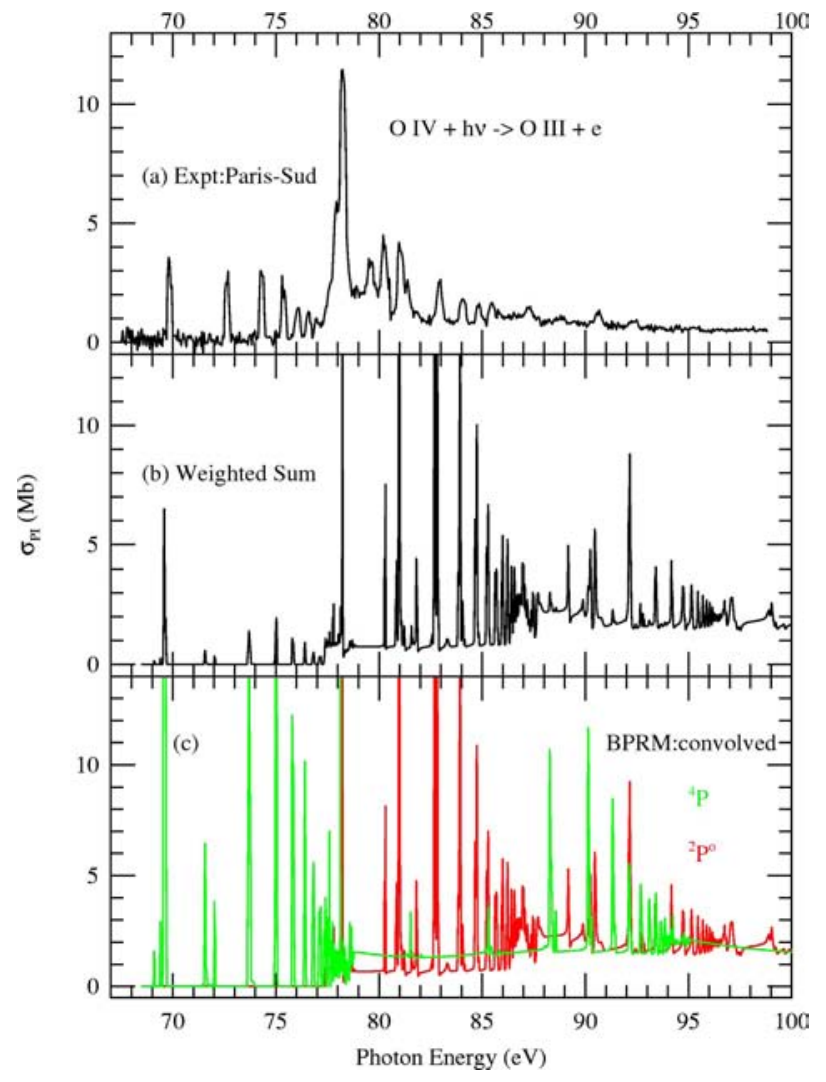

FIG. 6. (Color online) Comparison of photoionization cross sections $\sigma_{P I}$ of $\mathrm{O}$ IV with experiment: (c) red and green curves are statistical sum of convolved cross sections of levels ${ }^{2} P_{1 / 2,3 / 2}^{o}$ and ${ }^{4} P_{1 / 2,3 / 2,5 / 2}$ respectively; (b) weighted sum of the cross sections; (c) measured cross sections at University of Paris-Sud by Champeaux et al. [8].

possible reason could be the effect of correlations of configuration interactions for the ${ }^{4} P$ symmetry. For the rest of the resonances the measured positions agree with the theoretical ones. One exception is the measured resonance at about $79.5 \mathrm{eV}$ which is not found either in the ${ }^{2} P^{o}$ or the ${ }^{4} P$ calculated cross sections.

The calculated peaks of resonances in Fig. 6 are seen mostly higher than the measured ones. These peaks may be reduced via radiative decays, or dielectronic recombination, if the radiative rates are comparable to autoionization rates. However, the radiative decay rates of the relevant excited core thresholds $\left(\approx 10^{8}, 10^{9} \mathrm{sec}^{-1}\right)$ of $\mathrm{O} \mathrm{V}$ are several orders of magnitude lower than typical autoionization rate $\left(\approx 10^{13}-10^{14} \mathrm{sec}^{-1}\right)$, and thus should cause only little damping, i.e., reduction in peak values. As mentioned earlier, the calculated peaks are affected by resolution or different energy meshes, Gaussian energy width, and percentage abundances of states in the beam. Particular mixture fractions can even eliminate resonances, as seen in the comparison between Figs. 6(b) and 6(c). Number of resonances in convolved cross sections in Fig. 6(c) are wiped out in the weighted sum in Fig. 6(b). Hence, for identification of resonances, the experimental spectrum should be compared with the pure convolved cross sections, i.e., with Fig. 6(c), and as identified in Ref. [8]. 
The most significant feature in the measured cross sections is the prominent resonance at about $78 \mathrm{eV}$ [Fig. 6(a)]. This is a combined effect from the near-threshold resonances of the ${ }^{2} P^{o}$ ground state [Figs. 5(a) and 5(b)] and converging resonances of ${ }^{4} P$ levels to core threshold ${ }^{3} P_{1 / 2}^{o}$ [Figs. $5(\mathrm{c})-5(\mathrm{e})]$. Although narrow resonances are not often observed because of low strengths, the energy position indicate that the metastable ${ }^{4} P$ photoionization [green curve in Fig. $6(\mathrm{c})]$ is making a contribution to the feature. Theoretical resonances are resolved with a much finer energy mesh than the experimental beamwidth, and hence appear sharper. The Gaussian convolution does not broaden the features as in the experiment. Also the experimental beam distribution function is not in general pure Gaussian. In addition, the FWHM may not remain constant at all energies; a convolved peak can be flattened somewhat with a larger bandwidth. However, the fact that the prominent feature observed at $78 \mathrm{eV}$ can be explained in terms of contributions from ${ }^{2} P^{o}$ and ${ }^{4} P$ states is of potential importance in the determination of fractional abundances of the ground and excited states in the ion source. The difference in the background cross sections is an indication that the mixtures of states may not be exactly the same as quoted in the experiment. The background cross section can be reduced or enhanced in different energy regions by taking various fractions. The fractions $91 \%$ and $9 \%$ used in the present simulation may not exact match the experimental ones.

Comparison between the theoretical and experimental photoionization cross sections in Fig. 6 highlights two of the main points in this work: (i) a mixture of the ground ${ }^{2} P^{o}$ and metastable ${ }^{4} P$ states in the experimental beam represents the observed features, both theory and experiment show the same resonances, and (ii) the detailed cross sections depend on the fractional mixture of ionic states.

\section{Photoionization cross sections of $\mathrm{O} \mathrm{v}$}

Photoionization cross sections of $\mathrm{O} v$ are obtained for the ground $2 s^{2}\left({ }^{1} S\right)$ and the excited $2 s 2 p\left({ }^{3} P^{o},{ }^{1} P^{o}\right)$ states. The detailed features in $\sigma_{P I}$ are shown in Figs. 7(a)-7(c): ground state, ${ }^{1} S$-red in Fig. 7(c), ${ }^{3} P^{o}$-blue in Fig. 7(b), ${ }^{1} P^{o}$ - green in Fig. 7(a). Contrary to overlapping resonances of states for the other ions, $\mathrm{OV}$ resonances for the three states are almost in separated energy regions making it easy to distinguish the resonance complexes.

The detailed cross sections of three states are convolved with experimental bandwidth $(25 \mathrm{meV})$ at the University of Paris-Sud [8]. The convolved cross sections are presented in Fig. 7(d), where the original colors are kept for each state for easy identification. For comparison with experimental spectrum in Fig. 7(f), these convolved cross sections are summed with weighting factors $75 \%$ for ${ }^{1} S, 23 \%$ for ${ }^{3} P^{o}$, and $2 \%$ for ${ }^{1} P^{o}$, which are different from those quoted in the experiment. The fractions in the experimental beam as reported in Ref. [8] are 65\% for the ground state ${ }^{1} S$ and $35 \%$ for excited ${ }^{3} P^{o}$ state. The reason for inclusion of $2 s 2 p\left({ }^{1} P^{o}\right)$ cross sections in present theoretical results is the similarity of observed and calculated features in the low-energy region below $103.6 \mathrm{eV}$. Although the measured features in the cross sections below $103.6 \mathrm{eV}$, the ionization threshold for $2 s p 2\left({ }^{3} P^{o}\right)$ state, ap-

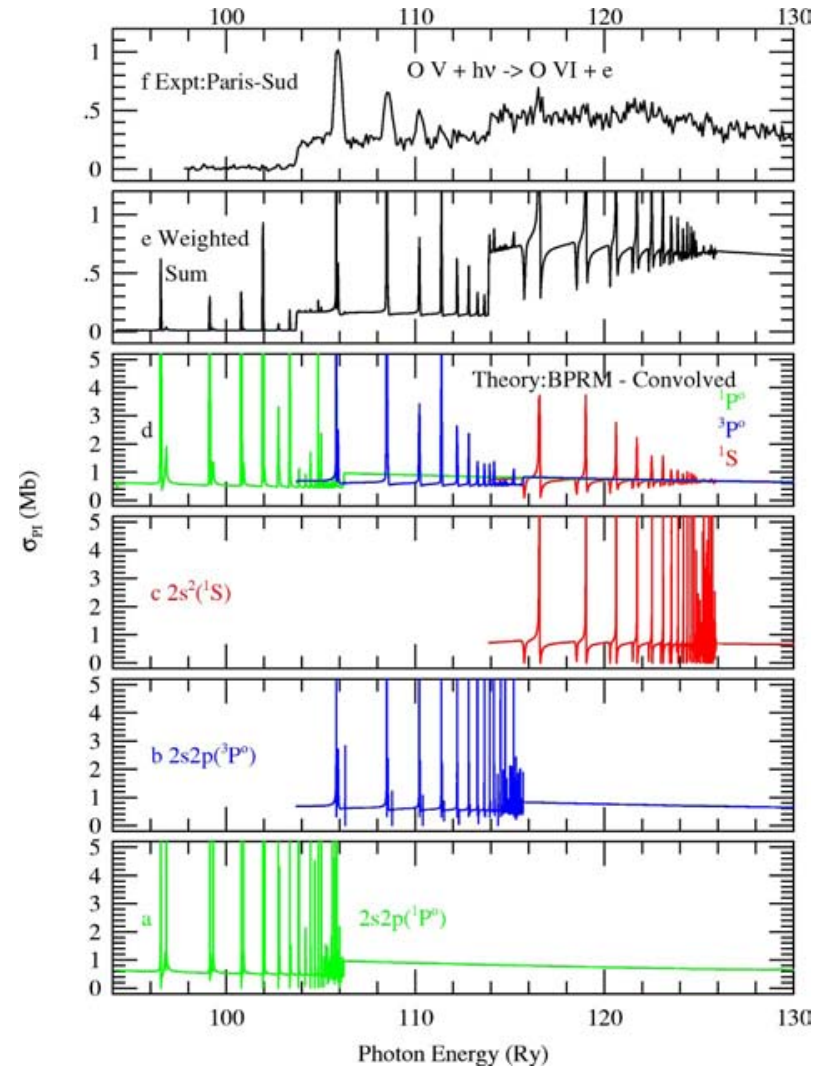

FIG. 7. (Color online) Photoionization cross sections $\sigma_{P I}$ of O v: panel (c), detailed $\sigma_{P I}$ of ground $2 s^{2}\left({ }^{1} S\right)$; (b),(a) excited $2 s 2 p\left({ }^{3} P^{o},{ }^{1} P^{o}\right)$ states; (d) convolved cross sections, (e) weighted sum of convolved cross sections; (f) measured cross sections at the University of Paris-Sud by Champeaux et al. [8].

pear as small kinks in Fig. 7(f), they do correspond to the resonances appearing at the same energy positions of $2 s 2 p\left({ }^{1} P^{o}\right)$ state of $\mathrm{O} \mathrm{v}$.

A clear rise seen in the background cross sections at about $103.6 \mathrm{eV}$ corresponds to the ${ }^{3} P^{o}$ ionization threshold, and at about $113.85 \mathrm{eV}$ to ${ }^{1} S$ ionization threshold, as noted both in Figs. 7(e) and 7(f). However, some differences in the enhancement of the background cross sections can be noted. Since present mixture includes higher percentage of the ${ }^{1} S$ ground state, a higher enhancement is expected in the weighted average. The calculated resonant peaks are also higher than the observed ones. The reason is the higher resolution in the calculations compared to the measurement as discussed in sections for other ions. However, the main objective is met, that is, all observed resonant features, shapes, and energy positions are reproduced in the calculated spectra. Identifications of the resonances are given in Ref. [8].

\section{CONCLUSION}

Photoionization cross section of oxygen in several stages of ionization has been obtained for the ground and low-lying excited states. These cross sections are compared with measured cross sections at three different experimental setups.

While the experimental measurement includes combined features of resonances belonging to different Rydberg series 
of states, and of a mixture of different states, theoretical work can evaluate them for individual states for identifications and fractional abundances.

Certain features in the measured cross sections are not seen prominently or are missing in previous calculations. The present paper shows that the missing features can be found with (i) higher resolution of resonances and (ii) inclusion of relativistic fine-structure effects. For most cases of this relatively low $\mathrm{Z}$ element, $L S$ coupling calculations can be adequate. However, for some metastable states, fine structure is crucial for the low-energy resonances which are allowed only via couplings of fine-structure levels. It is the fine-structure components, rather than the relativistic effects, that are important for the formation of these high peak resonances with nearly zero background. Inclusion of relativistic effects is certainly the more exact and accurate way to carry out the calculations.

\section{ACKNOWLEDGMENTS}

I would like to thank Anil Pradhan for discussions and suggestions. This work was partially supported by the National Science Foundation and the NASA Astrophysical Theory Program. The computational work was carried out at the Ohio Supercomputer Center.
[1] The Opacity Project $1 \& 2$, compiled by the Opacity Project (Institute of Physics, London, 1995,1996).

[2] D. G. Hummer, K. A. Berrington, W. Eissner, A. K. Pradhan, H. E. Saraph, and J. A. Tully, Astron. Astrophys. 279, 298 (1993).

[3] H. Kjeldsen, F. Folkmann, J. E. Hensen, H. Knudsen, M. S. Rasmussen, J. B. West, and T. Andersen, Astron. Astrophys. 524, L143 (1999).

[4] J.-M. Bizau, J.-M. Esteva, D. Cubaynes, F. J. Wuilleumier, C. Blancard, A. Compant La Fontaine, C. Couillaud, J. Lachkar, R. Marmoret, C. Remond, J. Bruneau, D. Hitz, P. Ludwig, and M. Delaumay, Phys. Rev. Lett. 84, 435 (2000).

[5] A. M. Covington, A. Aguilar, I. R. Covington, M. Gharailbeh, C. A. Shirley, R. A. Phaneuf, I. Alvarez, C. Cisneros, G. Hinojosa, J. D. Bozek, I. Dominguez, M. M. Sant'Ama, A. S. Schlachter, N. Berrah, S. N. Nahar, and B. M. McLaughlin, Phys. Rev. Lett. 87, 243002 (2001).

[6] A. Aguilar, A. M. Covington, G. Hinojosa, R. A. Phaneuf, I. Alvarez, C. Cisneros, J. D. Bozek, M. Sant'Ama, A. S. Schlachter, I. Dominguez, S. N. Nahar, and B. M. McLaughlin, Astrophys. J., Suppl. 146, 467 (2003).

[7] H. Kjeldsen, B. Kristensen, R. L. Brooks, F. Folkmann, H. Knudsen, and T. Andersen, Astrophys. J., Suppl. Ser. 138, 219
(2002).

[8] J.-P. Champeaux, J.-M. Bizau, D. Cubaynes, C. Blancard, S. Nahar, D. Hitz, J. Bruncau, and F. J. Wuilleumier, Astrophys. J. 148, 583 (2003).

[9] S. N. Nahar, Phys. Rev. A 58, 3766 (1998).

[10] S. N. Nahar and A. K. Pradhan, Phys. Rev. Lett. 68, 1488 (1992).

[11] H. L. Zhang, S. N. Nahar, and A. K. Pradhan, J. Phys. B 32, 1459 (1999).

[12] A. K. Pradhan, G. X. Chen, S. N. Nahar, and H. L. Zhang, Phys. Rev. Lett. 87, 183201 (2001).

[13] S. N. Nahar, Phys. Rev. A 65, 052702 (2002).

[14] M. J. Seaton, J. Phys. B 20, 6363 (1987).

[15] K. A. Berrington, P. G. Burke, K. Butler, M. J. Seaton, P. J. Storey, K. T. Taylor, and Yu. Yan, J. Phys. B 20, 6379 (1987).

[16] N. S. Scott and K. T. Taylor, Comput. Phys. Commun. 25, 347 (1982); N. S. Scott and P. G. Burke, J. Phys. B 12, 4299 (1980).

[17] K. A. Berrington, W. Eissner, and P. H. Norrington, Comput. Phys. Commun. 92, 290 (1995).

[18] W. Eissner, M. Jones, and N. Nussbaumer, Comput. Phys. Commun. 8, 270 (1974). 